BULL. AUSTRAL. MATH. SOC.

VOL. $12(1975), 337-350$.

\title{
On some integrodifferential equations in Banach spaces
}

\section{B.G. Pachpatte}

This paper is concerned with the stability, boundedness, and asymptotic behavior of solutions of integrodifferential systems of the form

$$
x^{\prime}(t)=A(t) x(t)+f\left(t, x(t), \int_{t_{0}}^{t} k(t, s, x(s)) d s\right) .
$$

We shall also investigate the behavioral relationships between the solutions of two integrodifferential systems related to this system.

\section{Introduction}

Let $B$ be a real Banach space. We are interested in the operators which are defined on subsets of $B$ and take values belonging to $B$. The operator $A$ is linear if it is additive and homogeneous. Denote its domain of definition by $D[A]$. The operator $A$ is said to be closed if the set of couples $(x, A x), x \in D[A]$, is closed in the topological product $B \times B$. For a real $\alpha$, suppose that $[I-\alpha A]^{-1}$ exists, where $I$ is the identity operator and $D\left[[I-\alpha A]^{-1}\right]$ is dense in $B$. Let $R(\alpha, A)=[I-\alpha A]^{-1}$ and since $A$ is closed we have

$$
\begin{aligned}
D[R(\alpha, A)] & =B, \\
{[I-\alpha A] R(\alpha, A) x } & =x, \quad(x \in B), \\
R(\alpha, A)[I-\alpha A] x & =x, \quad x \in D[A],
\end{aligned}
$$

Received 18 December 1974. 
where $R(\alpha, A)$ is called the resolvent of $A$. Let $J$ denote the interval $t_{0} \leq t<\infty, t_{0} \geq 0$, and $|\cdot|$ denote any suitable norm in $B$. We denote by $C[X, Y]$, the class of functions defined and continuous on $X$ taking values in $Y$, where $X$ and $Y$ are any convenient spaces. In this paper we wish to study the behavior of solutions of integrodifferential systems of the form

(1) $x^{\prime}(t)=A(t) x(t)+f\left(t, x(t), \int_{t_{0}}^{t} k(t, s, x(s)) d s\right), x\left(t_{0}\right)=x_{0}$, where $x \in B, f \in C[J \times B \times B, B]$ and $k \in C[J \times J \times B, B], f(t, 0,0) \equiv 0$, $k(t, s, 0) \equiv 0$, and the operator $A(t)$ satisfies the assumption (*) given below.

(*) Suppose that for $t \in J,\{A(t)\}$ is a one parameter family of closed linear operators. Assume that for each $t \in J$, the resolvent set of $A(t)$ includes all positive real numbers, that the domain of $R(\alpha, A(t))$ is dense in $B$, and that the domain $D[A(t)]$ of $A(t)$ is independent of $t$.

Let $x(t) \in D[A(t)]$ be a strongly continuous function defined for $t \in J$ and having a strong derivative $x^{\prime}(t)$ in $B$. We shall call $x(t)$ a solution of the integrodifferential system (1) if it satisfies equation (1), with the initial value $x\left(t_{0}\right)=x_{0}, x_{0} \in D\left[A\left(t_{0}\right)\right], t_{0} \geq \cdot 0$ for $t_{0}<t<\infty$.

In recent years differential equations in abstract spaces with operator coefficients have been studied by a number of authors. Since several problems for partial differential equations and integrodifferential equations can be reduced to problems for equations with unbounded operator coefficients in a suitable abstract space, the study of abstract differential equations is becoming increasingly important. Many recent papers have dealt with the existence and uniqueness of solutions of some integral and integrodifferential equations in Banach spaces; see, for example, Friedman and Shinbrot [1], Lovelady [5], [6], Marti [7], to mention a few. However, it often happens that once existence and uniqueness have been established a quite different analysis is required for finding more detailed boundedness and asymptotic properties of the 
solution. In this paper, we shall assume, without further mention, that (1) has a. solution with the initial value $x\left(t_{0}\right)=x_{0}, x_{0} \in D\left[A\left(t_{0}\right)\right]$, $t_{0} \geq 0$, and limit our discussion to some problems in stability, boundedness and asymptotic behavior of solutions of ( 1 ) and the integrodifferential systems related to this system. The integrodifferential systems considered in this paper contain, in particular, the abstract differential systems studied by Lakshmikantham [2], Lakshmikantham and Leela [4, Chapter 12], and the abstract integrodifferential systems recently studied by this author in [9], [11], by using different techniques.

\section{Main results}

In this section, theorems are stated and proved which yield conditions for stability, boundedness, and asymptotic behavior of the solutions of (1). We require the following integral inequality recently established by this author in [10].

LEMMA 1. Let $u(t), p(t)$, and $q(t)$ be real-valued nonnegative continuous functions defined on $J$, for which the inequality

$$
u(t) \leq u_{0}+\int_{t_{0}}^{t} p(s) u(s) d s+\int_{t_{0}}^{t} p(s)\left(\int_{t_{0}}^{s} q(\tau) u(\tau) d \tau\right) d s, t \in J,
$$

holds, where $u_{0}$ is a nonnegative constant. Then

$$
u(t) \leq u_{0}\left[1+\int_{t_{0}}^{t} p(s) \exp \left(\int_{t_{0}}^{s}(p(\tau)+q(\tau)) d \tau\right) d s\right], \quad t \in J
$$

In this section our interest lies in the following definitions.

DEFINITION 1. The solution $x=0$ of (1) is said to be exponentially asymptotically stable, if there exist positive constants $M$ and $\alpha$ such that for any solution $x(t)$ of $(1), x\left(t_{0}\right)=x_{0}$, the inequality

$$
|x(t)| \leq M\left|x_{0}\right| e^{-\alpha\left(t-t_{0}\right)}, \quad t \geq t_{0},
$$

holds for $\left|x_{0}\right|$ sufficiently small. 
DEFINITION 2. The solution $x=0$ of (I) is said to be uniformly slowly growing if, and only if, for every $\alpha>0$ there exists a constant $M$, possibly depending on $\alpha$, such that for any solution $x(t)$ of (1), $x\left(t_{0}\right)=x_{0}$, the inequality

$$
|x(t)| \leq M\left|x_{0}\right| e^{\alpha\left(t-t_{0}\right)}, t \geq t_{0},
$$

holds for $\left|x_{0}\right|<\infty$.

Our first theorem deals with the boundedness of the solutions of (1) under some suitable conditions on the functions involved in (1).

THEOREM 1. Assome that for each $t \in J, x \in B$,

$$
\lim _{h \rightarrow 0^{+}} R(h, A(t)) x=x \text {; }
$$

and

$$
\begin{gathered}
|R(h, A(t)) x+h f(t, x, z)| \leq|x|+h p(t)[|x|+|z|], \\
|k(t, s, x)| \leq q(s)|x|, 0 \leq s \leq t<\infty,
\end{gathered}
$$

for every $x, z \in B$ and for all sufficiently small $h>0$, where $p, q \in C\left[J, R^{+}\right]$and

$$
\int_{t_{0}}^{\infty} p(s) d s<\infty, \int_{t_{0}}^{\infty} q(s) d s<\infty \text {. }
$$

Then any solution $x(t)$ of (1) with $x\left(t_{0}\right)=x_{0}$ is bounded on $J$.

Proof. Let $x(t)$ be any solution of (1) with $x\left(t_{0}\right)=x_{0}$, and define $m(t)=|x(t)|$. For small $h>0$, we have

(6) $m(t+h) \leq\left|x(t+h)-R(h, A(t)) x(t)-h\left[f\left(t, x(t), \int_{t_{0}}^{t} k(t, s, x(s)) d s\right)\right]\right|$

$$
+\left|R(h, A(t)) x(t)+h\left[f\left(t, x(t), \int_{t_{0}}^{t} k(t, s, x(s)) d s\right)\right]\right| \text {. }
$$

Since for every $x \in D[A(t)], R(h, A(t))(I-h A(t)) x=x$, it follows that

$$
R(h, A(t)) x=x+h A(t) x+h[R(h, A(t)) A(t) x-A(t) x] .
$$


From (6), (7), together with (1), (2), (3), (4), ws obtain the inequality

$$
m^{\prime}(t) \leq p(t)\left[m(t)+\int_{t_{0}}^{t} q(s) m(s) d s\right] .
$$

Integrating both sides of (8) from $t_{0}$ to $t$, we get

$$
m(t) \leq m\left(t_{0}\right)+\int_{t_{0}}^{t} p(s) m(s) d s+\int_{t_{0}}^{t} p(s)\left(\int_{t_{0}}^{s} q(\tau) m(\tau) d \tau\right) d s .
$$

Now an application of Lemma 1 yields

$$
|x(t)| \leq\left|x_{0}\right|\left[1+\int_{t_{0}}^{t} p(s) \exp \left(\int_{t_{0}}^{s}(p(\tau)+q(\tau)) d \tau\right) d s\right] \text {. }
$$

The above estimation in view of the assumptions on $p$ and $q$ implies the boundedness of the solution $x(t)$ of (1) on $J$. This proves the theorem.

REMARK 1. It is important to note that Theorem 1 implies not only the boundedness, but the stability of $x(t)$, if $\left|x_{0}\right|$ is small enough. However, the above estimation does not prove the asymptotic stability.

Our next theorem shows that under some suitable conditions on the functions involved in ( 1 ), any solution of (1) is exponentially asymptotically stable.

THEOREM 2. Assume that for each $t \in J, x \in B$,

$$
\lim _{h \rightarrow 0^{+}} R(h, A(t)) x=x \text {, }
$$

and

$$
\begin{gathered}
|R(h, A(t)) x+h f(t, x, z)| \leq|x|+h e^{-\alpha t} p(t)[|x|+|z|], \\
|k(t, 8, x)| \leq e^{-\alpha t} q(s)|x|, 0 \leq s \leq t<\infty,
\end{gathered}
$$

for every $x, z \in B$, for a constant $\alpha \geq 1$, and for alz sufficiently small $h>0$, where $p, q \in C\left[J, R^{+}\right], p(t) \geq 1$, and

$$
\int_{t_{0}}^{\infty} p(s) d s<\infty, \int_{t_{0}}^{\infty} e^{-\alpha s} q(s) d s<\infty .
$$


Then any solution $x(t)$ of (1) with $x\left(t_{0}\right)=x_{0}$ is exponentially asymptotically stable.

Proof. Let $x(t)$ be any solution of (1) with $x\left(t_{0}\right)=x_{0}$, and define $m(t)=|x(t)|$. Now following a similar argument as in the proof of Theorem 1 we obtain the inequality

$$
m^{\prime}(t) \leq e^{-\alpha t} p(t)\left[m(t)+e^{-\alpha t} \int_{t_{0}}^{t} q(s) m(s) d s\right]
$$

Multiplying both sides of (13) by $e^{\alpha t}$ and then adding $\alpha e^{\alpha t} m(t)$, we have

$$
\begin{aligned}
{\left[e^{\alpha t} m(t)\right]^{\prime} } & \leq \alpha e^{\alpha t} m(t)+p(t)\left[m(t)+e^{-\alpha t} \int_{t_{0}}^{t} q(s) m(s) d s\right] \\
& \leq \alpha p(t) m(t) e^{\alpha t}+\alpha p(t) e^{\alpha t}\left[m(t)+e^{-\alpha t} \int_{t_{0}}^{t} q(s) m(s) d s\right],
\end{aligned}
$$

since $p(t) \geq 1, \alpha \geq 1, e^{\alpha t} \geq 1$. Integrating both sides of the above inequality from $t_{0}$ to $t$, we get

$$
\begin{aligned}
m(t) e^{\alpha t} \leq m\left(t_{0}\right) e^{\alpha t_{0}}+\int_{t_{0}}^{t} 2 \alpha p(s) m(s) e^{\alpha s} d s & \\
& +\int_{t_{0}}^{t} 2 \alpha p(s)\left(\int_{t_{0}}^{s} \frac{z_{2}}{2} q(\tau) e^{-\alpha \tau} m(\tau) e^{\alpha \tau} d \tau\right) d s .
\end{aligned}
$$

Now, applying Lemma I. with $u(t)=m(t) e^{\alpha t}$, and then multiplying by $e^{-\alpha t}$, we obtain

$$
|x(t)| \leq\left|x_{0}\right| e^{-\alpha\left(t-t_{0}\right)}\left[1+\int_{t_{0}}^{t} 2 \alpha p(s) \exp \left(\int_{t_{0}}^{s}\left(2 \alpha p(\tau)+\frac{1}{2} q(\tau) e^{-\alpha \tau}\right) d \tau\right) d s\right] .
$$

The above estimation in view of the assumptions on $p$ and $q$ implies

$$
|x(t)| \leq M\left|x_{0}\right| e^{-\alpha\left(t-t_{0}\right)}, t \geq t_{0},
$$

where $M>0$ is a constant. This proves the exponential asymptotic 
stability of the solution $x(t)$ of (1).

Our Theorem 3 below demonstrates that the solution of (1) grows more slowly than any positive exponential.

THEOREM 3. Assume that for each $t \in J, x \in B$,

$$
\lim _{h \rightarrow 0^{+}} R(h, A(t)) x=x \text {, }
$$

and

$$
\begin{gathered}
|R(h, A(t)) x+h f(t, x, z)| \leq|x|+h e^{\alpha t} p(t)[|x|+|z|], \\
|k(t, s, x)| \leq e^{\alpha t} q(s)|x|, \quad 0 \leq s \leq t<\infty,
\end{gathered}
$$

for every $x, z \in B$, for a constant $\alpha>0$, and for all sufficiently small $h>0$, where $p, q \in C\left[J, R^{+}\right]$and

$$
\int_{t_{0}}^{\infty} e^{\alpha s} p(s) \exp \left(\int_{t_{0}}^{s} e^{\alpha \tau}[p(\tau)+q(\tau)] d \tau\right) d s \leq N,
$$

where $N>0$ is a constant. Then any solution $x(t)$ of (1) with $x\left(t_{0}\right)=x_{0}$ is uniformly slowly growing.

Proof. Let $x(t)$ be any solution of (1) with $x\left(t_{0}\right)=x_{0}$, and define $m(t)=|x(t)|$. Now following a similar argument as in the proof of Theorem 1 we obtain the inequality

$$
m^{\prime}(t) \leq e^{\alpha t} p(t)\left[m(t)+e^{\alpha t} \int_{t_{0}}^{t} q(s) m(s) d s\right] .
$$

Multiplying both sides of the above inequality by $e^{-\alpha t}$, and then subtracting $e^{-a t} m(t)$, we have

$$
\begin{aligned}
{\left[m(t) e^{-\alpha t}\right]^{\prime} } & \leq-\alpha e^{-\alpha t} m(t)+p(t)\left[m(t)+e^{\alpha t} \int_{t_{0}}^{t} q(s) m(s) d s\right] \\
& \leq e^{\alpha t} p(t)\left[m(t) e^{-\alpha t}+\int_{t_{0}}^{t} q(s) e^{\alpha s} m(s) e^{-\alpha s} d s\right],
\end{aligned}
$$

since $e^{\alpha t} \geq 1$. Integrating both sides of the above inequality from $t_{0}$ 
to $t$, we get

$$
\begin{aligned}
m(t) e^{-\alpha t} \leq m\left(t_{0}\right) e^{-\alpha t} 0 & \int_{t_{0}}^{t} e^{\alpha s} p(s) m(s) e^{-\alpha s} d s \\
& +\int_{t_{0}}^{t} e^{\alpha s} p(s)\left(\int_{t_{0}}^{s} e^{\alpha \tau} q(\tau) m(\tau) e^{-\alpha \tau} d \tau\right) d s .
\end{aligned}
$$

Now, applying Lemma 1 with $u(t)=m(t) e^{-\alpha t}$ and then multiplying by $e^{\alpha t}$, we obtain

$$
|x(t)| \leq\left|x_{0}\right| e^{\alpha\left(t-t_{0}\right)}\left[I+\int_{t_{0}}^{t} e^{\alpha s} p(s) \exp \left(\int_{t_{0}}^{s} e^{\alpha \tau}(p(\tau)+q(\tau)) d \tau\right) d s\right] .
$$

The above estimation in view of the assumption (17) implies

$$
|x(t)| \leq M\left|x_{0}\right| e^{\alpha\left(t-t_{0}\right)}, t \geq t_{0},
$$

where $M=1+N$. This proves that the solution of (1) is uniformly slowly growing.

REMARK 2. We note that the important special case which is covered by the integrodifferential system (1) (when the integral term in (1) is absent) is the abstract differential system studied by Lakshmikantham [2], and Lakshmikantham and Leela [4, Chapter 12].

\section{Behavioral relationships}

In this section we shall investigate the behavioral relationships between the solutions of integrodifferential systems

$$
\text { (18) } \begin{aligned}
x^{\prime}(t)=A(t) x(t)+f(t, x(t))+ & \int_{t_{0}}^{t} k(t, s, x(s)) d s \\
& +\int_{t_{0}}^{t} G(t, s, x(s)) d s, x\left(t_{0}\right)=x_{0},
\end{aligned}
$$

and

(19) $y^{\prime}(t)=A(t) y(t)+f(t, y(t))+\int_{t_{0}}^{t} k(t, s, y(s)) d s, y\left(t_{0}\right)=y_{0}$, 
where $x, y \in B, f \in C[J \times B, B], k, G \in C\left[J \times_{e} \times \times_{B}, B\right]$, and the operator $A(t)$ is as defined above.

To obtain our results in this section, we make use of the following lemma which can be found in [11]. We shall state it in a suitable form whose proof needs very little modification of the proof of the basic comparison theorem given in [3, Theorem 1.4.1]. For a similar lemma we refer the reader to Nohel [8].

LEMMA 2. Let the scalar functions $W_{I} \in C[J \times B, B]$, $W_{2} \in C[J \times J \times B, B]$ and assume $W_{2}(t, s, r)$ is nondecreasing in $r$ for each $t, s \in J$. Suppose that $r(t)$ is the solution of the scalar integrodifferential equation

(20) $r^{\prime}(t)=W_{1}(t, r(t))+\int_{t_{0}}^{t} W_{2}\left(t, s, r(s)+\psi(s) e^{\alpha s}\right) d s$,

$$
\alpha>0, \quad r\left(t_{0}\right)=r_{0},
$$

existing to the right of $t_{0}$, where $\psi(t) \geq 0$ is a continuous franction defined on $J$. Let $m(t) \geq 0$ be continuous on $J$ such that $m\left(t_{0}\right) \leq r_{0}$ and let it satisfy

$$
m^{\prime}(t) \leq W_{1}(t, m(t))+\int_{t_{0}}^{t} w_{2}\left(t, s, m(s)+\psi(s) e^{\alpha s}\right) d s,
$$

where $m^{\prime}(t)=\underset{h \rightarrow 0^{+}}{\lim \sup } \frac{1}{h}[m(t+h)-m(t)]$. Then

$$
m(t) \leq r(t), \quad t \in J .
$$

We shall now give definitions of various types of stability which will be discussed in this section.

Let $x(t)$ and $y(t)$ be any two solutions of the integrodifferential systems (18) and (19) respectively.

DEFINITION 3. The system (18) or (19) is said to be equi-stable with respect to the system (19) or (18), if for each $\varepsilon>0$ and $t_{0} \geq 0$, there exists a positive function $\eta\left(t_{0}, \varepsilon\right)$ continuous in $t_{0}$ for each $\varepsilon$, such that 


$$
|x(t)-y(t)|<\varepsilon
$$

whenever $\left|x_{0}-y_{0}\right| \leq n\left(t_{0}, \varepsilon\right)$ for $t \geq t_{0}$.

DEFINITION 4.. The system (18) or (19) is said to be quasi-equiasymptotically stable with respect to (19) or (18) if for each $\varepsilon>0$, $\alpha>0$ and $t_{0} \geq 0$, there exists a positive number $T\left(t_{0}, \varepsilon, \alpha\right)$, such that

$$
|x(t)-y(t)|<\varepsilon
$$

whenever $\left|x_{0}-y_{0}\right| \leq \alpha^{\prime}$ for $t \geq t_{0}+T\left(t_{0}, \varepsilon, \alpha\right)$.

DEFINITION 5. If Definitions 3 and 4 hold simultaneously, then the system (18) or (19) is said to be equi-asymptotically stable with respect to the system (19) or (18).

REMARK 3. We note that if $n\left(t_{0}, \varepsilon\right)=n(\varepsilon)$ and $T\left(t_{0}, \varepsilon, \alpha\right)=T(\varepsilon, \alpha)$, that is, if they are independent of $t_{0}$, equi in the above definitions will be replaced by uniform.

Theorem 4 below establishes the asymptotic relationship between the solutions of (18) and (19).

THEOREM 4. Let the scalar functions $W_{1}, W_{2}$ be as defined in Lemma 2. Let $r(t)$ be any bounded solution of (20) existing to the right of $t_{0}$. Assume that for each $t \in J, x, y \in B$, and

$$
\lim _{h \rightarrow 0} R(h, A(t))(x-y)=x-y \text {. }
$$

and

$$
\left|R(h, A(t))(x-y)+h\left\{\int_{t_{0}}^{t}[k(t, s, x)-k(t, s, y)] d s\right\}\right| \leq|x-y|(1-\alpha h),
$$

$$
\begin{gathered}
|f(t, x)-f(t, y)| \leq w_{1}\left(t,|x-y| e^{\alpha t}\right) e^{-\alpha t}, \\
|G(t, s, x)| \leq w_{2}\left(t, s,|x| e^{\alpha s}\right) e^{-\alpha t},
\end{gathered}
$$

for every $x, y \in B$, and for all sufficiently small $h>0$. Then

$$
\lim _{h \rightarrow \infty}|x(t)-y(t)|=0 \text {, }
$$


where $x(t)$ and $y(t)$ are the solutions of (18) and (19) respectively for all $t \geq t_{0}$ such that $\left|x_{0}-y_{0}\right| \leq x_{0}$.

Proof. Define $m(t)=e^{\alpha t}|x(t)-y(t)|$. For small $h>0$ we have (25)

$$
\begin{aligned}
m(t+h) \leq & e^{\alpha(t+h)}[\mid x(t+h)-R(h, A(t)) x(t)-h\{f(t, x(t)) \\
& \left.+\int_{t_{0}}^{t} k(t, s, x(s)) d s+\int_{t_{0}}^{t} G(t, s, x(s)) d s\right\}-\{y(t+h) \\
& -R(h, A(t)) y(t)\}-h\left\{f(t, y(t))+\int_{t_{0}}^{t} k(t, s, y(s)) d s\right\} \mid \\
& +\mid R(h, A(t)) x(t)-R(h, A(t)) y(t)+h\{f(t, x(t))-f(t, y(t))\} \\
& \left.+h\left\{\int_{t_{0}}^{t}[k(t, s, x(s))-k(t, s, y(s))] d s+\int_{t_{0}}^{t} G(t, s, x(s)) d s\right\} \mid\right] .
\end{aligned}
$$

Since $x \in D[A(t)], R(h, A(t))(I-h A(t)) x=x$, it follows that

$$
R(h, A(t)) x=x+h A(t) x+h[R(h, A(t)) A(t) x-A(t) x] .
$$

Similarly for every $y \in D[A(t)], R(h, A(t))(I-h A(t)) y=y$, it follows that

$$
R(h, A(t)) y=y+h A(t) y+h[R(h, A(t)) A(t) y-A(t) y] .
$$

From (25), (26), (27) together with (18), (19), (21), (22), (23), (24) we obtain the inequality

(28) $m^{\prime}(t) \leq w_{1}(t, m(t))+\int_{t_{0}}^{t} w_{2}\left(t, s, m(s)+\psi(s) e^{\alpha s}\right) d s$,

where $\psi(t)=|y(t)|$. Now applying Lemme 2 to (20) and (28) we have

$$
e^{\alpha t}|x(t)-y(t)| \leq r(t), \quad t \in J \text {. }
$$

Since the solution $r(t)$ of (20) is bounded we can bring $e^{\alpha t}, \alpha>0$, in (29) to the other side; then we obtain

$$
\lim _{t \rightarrow \infty}|x(t)-y(t)|=0 \text {. }
$$

The proof of the theorem is complete. 
REMARK 4. The above theorem is an extension of a similar result due to Lakshmikantham [2, pp. 274-275] obtained for abstract differential systems. Further, we note that the integrodifferential systems considered in (1), (18), and (19) are of a more general type and contain, in particular, the integrodifferential systems recently studied by this author in $[9],[11]$.

Finally we state and prove the following theorem which yields sufficient conditions for various forms of stability in the sense of the Definitions 3, 4 and 5 .

THEOREM 5. Suppose that all assumptions of Theorem 4 hold, and that the identically zero solution of $(20)$ is equi-stable. Then, the integrodifferential systems (18) and (19) are quasi-equi-asymptotically and equi-asymptotically stable.

Proof. Suppose that the scalar integrodifferential equation (20) is equi-stable and assume that $x(t)$ and $y(t)$ are any two solutions of (18) and (19) such that $\left|x_{0}-y_{0}\right| \leq r_{0}$. Then, as in Theorem 4, we have

$$
e^{\alpha t}|x(t)-y(t)| \leq r(t), \quad t \in J \text {. }
$$

From (30) it is easy to see that the systems (18) and (19) satisfy Definition $I$.

Suppose $\left\{t_{n}\right\}$ is a divergent sequence and assume that

$$
\left|x\left(t_{n}\right)-y\left(t_{n}\right)\right| \geq \varepsilon_{1}
$$

where $\varepsilon_{1}$ is an arbitrary constant greater than zero. Then we obtain

$$
\varepsilon_{1} e^{\alpha t} n \leq r\left(t_{n}\right)<\varepsilon
$$

As $n \rightarrow \infty$, this leads to a contradiction, because $\varepsilon_{1}$ and $\varepsilon$ are positive. Since $\varepsilon_{1}$ is arbitrary this shows that the systems satisfy Definition 2. This completes the proof of the theorem.

REMARK 5. If the functions involved in Theorem 5 are independent of $t_{0}$ the equi will be replaced by uniform. 


\section{References}

[1] Avner Friedman and Marvin Shinbrot, "Volterra integral equations in Banach space", Trans. Amer. Math. Soc. 126 (1967), 131-179.

[2] V. Lakshmikantham, "Differential equations in Banach spaces and the extension of Lyapunov's method", Proc. Cambridge Philos. Soc. 59 (1963), 373-381.

[3] V. Lakshmikantham and S. Leela, Differential and integral inequalities: theory and applications. Volume I: Ordinary differential equations (Mathematics in Science and Engineering, 55-I. Academic Press, New York and London, 1969).

[4] V. Lakshmikantham and S. Leela, Differential and integral inequalities: theory and applications. Volume II: Frotional, partial, abstract, and complex differential equations (Mathematics in Science and Engineering, 55-II. Academic Press, New York and London, 1969).

[5] David Lowell Lovelady, "A functional differential equation in a Banach space", Fronkcial. Ekvac. 14 (1971), 111-122.

[6] David Lowell Lovelady, "A global existence theorem for a functional differential equation", An. Sti. Univ. "AZ. I. Cuza" Ia§i Sect. I a Mat. 18 (1972), 343-349.

[7] Jürg T. Marti, "On integro-differential equations in Banach spaces", Pacific J. Math. 20 (1967), 99-108.

[8] J.A. Nohel, "Some problems in nonlinear Volterra integral equations", Bulz. Amer. Math. Soc. 68 (1962), 323-329.

[9] B.G. Pachpatte, "Comparison theorems for integro-differential equations in Banach spaces", Proc. Nat. Acad. Sci. India 42(A) (1972), 125-128.

[10] B.G. Pachpatte, "A note on Gronwall-Bellman inequality", J. Math. Anal. App I. 44 (1973), 758-762. 
[11] B.G. Pachpatte, "On the stability and asymptotic hehavior of solutions of integrodifferential equations in Banach spaces", J. Math. Anal. App Z. (to appear).

Department of Mathematics,

Deogiri Col lege Aurangabad,

Maharashtra,

India. 